\title{
DYNAMIC CHARACTERISTICS OF COMMUNITY STRUCTURE AND SEASONAL VARIATION OF FISHERY SPECIES IN THE BOHAI SEA, CHINA
}

\author{
FORRUQ RAHMAN, M. ${ }^{1,3}-$ SHAN, X. J. ${ }^{2,3}-$ LIN, Q. ${ }^{3}-$ CHEN, Y. L. ${ }^{3}-$ MAMUN, A. A. ${ }^{4}-$ \\ BARMAN, P. P. ${ }^{1}-$ YANAN, H. $^{1}-$ LIU, Q. ${ }^{1 *}$ \\ ${ }^{1}$ College of Fisheries, Department of Marine Fisheries, Ocean University of China \\ Qingdao 266003, People's Republic of China \\ ${ }^{2}$ Function Laboratory for Marine Fisheries Science and Food Production Processes, Pilot \\ National Laboratory for Marine Science and Technology (Qingdao) \\ Qingdao 266237, People's Republic of China \\ ${ }^{3}$ Provincial Key Laboratory of Fishery Resources and Ecological Environment, Yellow Sea \\ Fisheries Research Institute, Chinese Academy of Fishery Sciences \\ Qingdao 266071, People's Republic of China \\ ${ }^{4}$ College of Marine Life Science, Department of Marine Ecology, Ocean University of China \\ Qingdao 266003, People's Republic of China \\ Corresponding author \\ e-mail:qunliu@mail.ouc.edu.cn; phone:+86-532-8203-1715
}

(Received $9^{\text {th }}$ Jul 2019; accepted $15^{\text {th }}$ Nov 2019)

\begin{abstract}
The structural patterns and the seasonal dynamics of fishery species were studied in the Bohai Sea, based on fishery-independent data surveyed in each season sampling stations. A total of 88 fishery species belonging to 64 families were recorded, in detail 43 fishes, 29 crustaceans, 12 molluscas, three echinoderms and one species of scyphozoan. Among them, 21 species commonly found throughout the study period defined as 'common' species, and eight species those were the highest contributors defined as 'dominant' species (based on SIMPER analysis, their cumulative contribution was more than 70\%). Maximum abundance was recorded in the summer season while species number was higher in both summer and autumn season. Among the community parameters, species richness (3.79), diversity (2.31) and Simpson indices (0.88) peaked in the autumn season whereas species evenness (0.95) was in the winter. However, species diversity, evenness, and Simpson indices were lower in the summer season $(0.57,0.18$ and 0.18 , respectively) while species richness was in the winter (1.65). Multivariate PCoA analysis revealed that there was a significant seasonal variation in fisheries assemblages. Furthermore, ANOSIM (Global $R=0.467, P=0.01$ ) indicated a distinct community structure of fishery species between the four seasons. Thus, this result suggests that fisheries assemblages might be influenced by the seasonal dynamics of the ecological condition of the Bohai Sea.
\end{abstract}

Keywords: fisheries assemblages, multivariate analyses, community parameters, seasonal patterns, trawl survey data

\section{Introduction}

The Bohai Sea, a semi-enclosed inland sea is a unique waterbody that houses rich marine and coastal biological resources (i.e., fishes/shellfishes; oil, gas, and minerals; estuaries; tidal flats; seagrass beds) as well as a great potentiality for mariculture and tourism purpose in the northeastern China (Zhang et al., 2002; Song and Duan, 2018). However, the rapid human 
settlement and industrial development that was triggered from 1985 along the coast of Bohai Sea accelerated the degradation in estuarine and marine ecosystems by altering hydropedological properties (i.e., water and sediments qualities) over the decades (Liu et al., 2011; Gao et al., 2014; Li et al., 2016). Besides, the changing climate variabilities such as temperature $(T)$, salinity $(S)$, and biogenic elements altered the Bohai Sea ecosystems which significantly influenced the living biotic communities (Lin et al., 2001; Yu et al., 2009). The variations of species in the communities are closely interrelated with the ecological conditions of water masses. Over the recent decades, anthropogenic activities mainly linked to urbanization and industrialization have a notable effect on the species diversity of the primary producers and benthos communities which may have triggered off the declining trend of fishery populations in the Bohai Sea (Hu et al., 2011; Liu et al., 2011).

Several studies have been focused on the characteristics of fish and fishery dynamics of the Bohai Sea (Chen et al., 1997; Tang et al., 2003; Jin, 2004; Shan et al., 2012, 2016; Zhang et al., 2012). Investigations have been reported that fishery stock status in the Bohai Sea experienced declining trends (Liu et al., 1990; Zhang et al., 2006; Zhou et al., 2013). Moreover, the Bohai Sea has been considered one of the most exploited fishing ground in the world (Wu et al., 2017; Liu et al., 2017). As a result, species abundance, diversity, and trophic structure had significantly shrunk in this ecosystem (Deng and Jin, 2001; Jin, 2004; Zhang and Tang, 2004; Shan et al., 2012). Spatio-temporal shifts of dominant species from largesize of high economic value to short-lived, low-trophic-level, low economic value and changes of ecosystem structure and functioning on the decadal-scale have been documented (Shan et al., 2013, 2016; Rahman et al., 2019).

The present study accounts the Bohai Sea as a major fishery center in China in view, and evaluated a one-year fishery-independent data (i.e., a pair of trawlers) to analyze the community patterns and dynamics of the fishery species. The specific aims of the research were (a) to document the taxonomic composition and species distribution patterns of fishery species, and (b) to illustrate the community patterns and the seasonal dynamics of fishery species during the study period.

\section{Materials and methods}

\section{Study area}

The Bohai Sea $\left(37^{\circ} 07^{\circ} \sim 41^{\circ} \mathrm{N}\right.$ and $\left.117^{\circ} 35^{\circ} \sim 121^{\circ} 10^{\circ} \mathrm{E}\right)$ is a semi-enclosed marginal sea of the northwestern Pacific Ocean on the northern coast of China. It extends for about $450 \mathrm{~km}$ from north to south and about $350 \mathrm{~km}$ from east to west, where the Bohai Strait is used as a junction to the Yellow Sea between Shandong and Liaodong Peninsulas (Fig. 1). It has an area of 77,000 km², which includes Bohai, Laizhou, and Liaodong Bays. The water depth varies at $10-20 \mathrm{~m}$ in the continental shelf where maximal water depth of $70 \mathrm{~m}$ is recorded in the northern part of Bohai Strait. The hydrographic conditions have greatly influenced by many factors such as river discharges, wind-tide-thermohaline circulation, stratification during summer, and vertical and horizontal mixing of nutrients in winter that support higher primary productivity (Ning et al., 2010). 


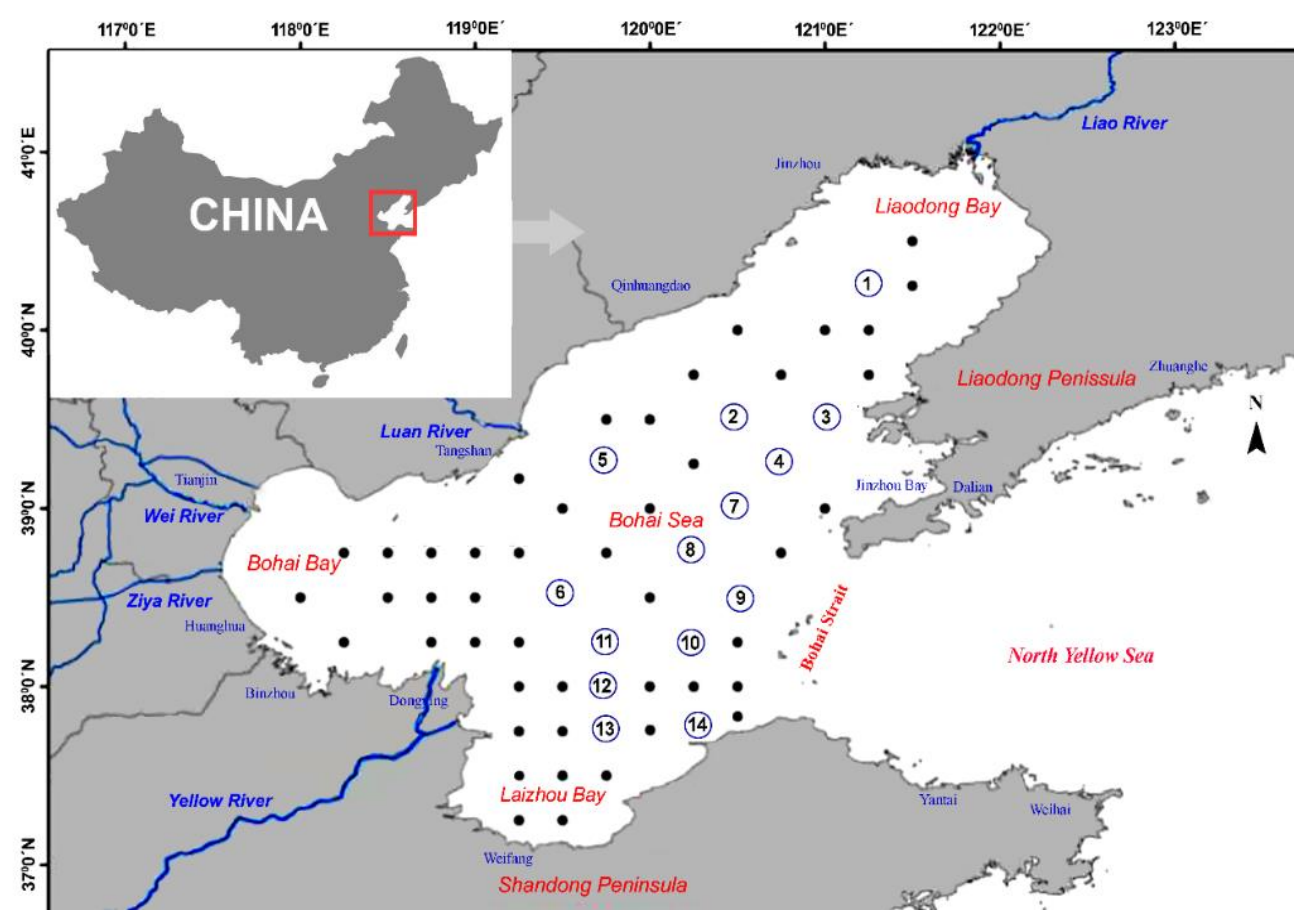

Figure 1. Geographical locations of sampling points in the Bohai Sea, China

\section{Data source}

Data were collected from a pair trawler (i.e., the capability was 200 horsepower; the distance between wings was $22.6 \mathrm{~m}$, headline height was between $5 \mathrm{~m}$ and $6 \mathrm{~m}$, cod mesh size was $2 \mathrm{~cm}$, and circumference was $30.6 \mathrm{~m}$.) survey carried out by the Yellow Sea Fisheries Research Institute. The surveyed sampling stations were designed on a fixed station grid of $0.5^{\circ} \mathrm{N} \times 0.5^{\circ} \mathrm{E}$. The hauls were lowered for sampling during the daytime, the duration at least 15 minutes to 60 minutes and an average hauling speed of $5.09 \mathrm{~km} / \mathrm{h}$. The average sampling depth was 23.18 meters (Table 1). All data were then standardized to a one-hour trawl duration for consistency. The survey period divided into four seasons namely as winter (January), spring (May), summer (August), and autumn (November) season. Samples were collected from the fixed 70 sampling stations. Among them, 14 stations were surveyed in each season. We selected these common sampling stations for analysis among the seasons.

Collected fishery specimens at each station were sorted firstly family and order level, then to species level, or the lowest possible taxon level. Subsequently, total abundance/individual abundance and species composition were recorded. The abundance of the species was expressed as the number of individual species catch per haul (ind./haul), and the composition of the species was expressed as a percentage of the total number of species recorded $(\%)$. 
Table 1. Position and characteristics (latitude, longitude, sampling site, aver. depth and aver. haul speed) of the sampling stations during the pair-trawl survey in the Bohai Sea

\begin{tabular}{|c|c|c|c|c|c|}
\hline \multirow{2}{*}{$\begin{array}{c}\text { Station SL } \\
\text { No. }\end{array}$} & \multicolumn{2}{|c|}{ Net placement } & \multirow{2}{*}{ Sampling site } & \multirow{2}{*}{$\begin{array}{c}\text { Average depth } \\
\text { (m) }\end{array}$} & \multirow{2}{*}{$\begin{array}{c}\text { Average haul speed } \\
(\mathrm{km} / \mathrm{h})\end{array}$} \\
\hline & Latitude & Longitude & & & \\
\hline 1 & $40^{\circ} 25^{\prime} \mathrm{N}$ & $121^{\circ} 25^{\prime} \mathrm{E}$ & Liaodong Bay & 23.6 & 5.19 \\
\hline 2 & $39^{\circ} 50^{\prime} \mathrm{N}$ & $120^{\circ} 50^{\prime} \mathrm{E}$ & Liaodong Bay & 25.9 & 5.06 \\
\hline 3 & $39^{\circ} 50^{\prime} \mathrm{N}$ & $121^{\circ} 00^{\prime} \mathrm{E}$ & Liaodong Bay & 25.2 & 4.39 \\
\hline 4 & $39^{\circ} 25^{\prime} \mathrm{N}$ & $120^{\circ} 75^{\prime} \mathrm{E}$ & Liaodong Bay & 25.73 & 4.87 \\
\hline 5 & $39^{\circ} 25^{\prime} \mathrm{N}$ & $119^{\circ} 75^{\prime} \mathrm{E}$ & Central Bohai & 25.8 & 5.24 \\
\hline 6 & $38^{\circ} 50^{\prime} \mathrm{N}$ & $119^{\circ} 50^{\prime} \mathrm{E}$ & Central Bohai & 25.83 & 5.0 \\
\hline 7 & $39^{\circ} 00^{\prime} \mathrm{N}$ & $120^{\circ} 50^{\prime} \mathrm{E}$ & Central Bohai & 24.57 & 5.56 \\
\hline 8 & $38^{\circ} 75^{\prime} \mathrm{N}$ & $120^{\circ} 25^{\prime} \mathrm{E}$ & Central Bohai & 25.83 & 5.13 \\
\hline 9 & $38^{\circ} 50^{\prime} \mathrm{N}$ & $120^{\circ} 50^{\prime} \mathrm{E}$ & Central Bohai & 28.43 & 5.43 \\
\hline 10 & $38^{\circ} 25^{\prime} \mathrm{N}$ & $120^{\circ} 25^{\prime} \mathrm{E}$ & Central Bohai & 23.93 & 4.95 \\
\hline 11 & $38^{\circ} 25^{\prime} \mathrm{N}$ & $119^{\circ} 75^{\prime} \mathrm{E}$ & Laizhou Bay & 22.67 & 4.82 \\
\hline 12 & $38^{\circ} 00^{\prime} \mathrm{N}$ & $119^{\circ} 75^{\prime} \mathrm{E}$ & Laizhou Bay & 16.73 & 5.69 \\
\hline 13 & $37^{\circ} 75^{\prime} \mathrm{N}$ & $119^{\circ} 75^{\prime} \mathrm{E}$ & Laizhou Bay & 15.33 & 5.13 \\
\hline 14 & $37^{\circ} 75^{\prime} \mathrm{N}$ & $120^{\circ} 25^{\prime} \mathrm{E}$ & Laizhou Bay & 14.93 & 4.82 \\
\hline
\end{tabular}

\section{Data analysis}

Diversity patterns of fishery species were summarized by frequently used diversity measuring indices such as Shannon-Wiener Index $\left(H^{\prime}\right)$ (Shannon \& Weaver) (Eq. 1), species evenness $\left(J^{\prime}\right)$ (Pielou) (Eq. 2), species richness $(d)$ (Margalef) (Eq. 3) and Simpson index (1- $\left.\lambda^{\prime}\right)$ (Simpson) (Eq. 4), these four indices were measured using the following formulas:

$$
\begin{gathered}
H^{\prime}=\sum_{i=1}^{S} P_{i\left(\ln P_{i}\right)} \\
J^{\prime}=H^{\prime} / \ln S \\
d=(S-1) / \ln N \\
1-\lambda^{\prime}=1-\operatorname{SUM}\left(N_{i} *\left(N_{i}-1\right)\right) /(N *(N-1))
\end{gathered}
$$

where, $H^{\prime}=$ observed diversity index; $P_{i}=$ proportion of the total count arising from the $i$ th species; $S=$ total number of species; and $N=$ the total number of individuals.

Both multivariate and univariate analyses were conducted for summarizing seasonal variation in fishery dynamics and their seasonal patterns of community structure using PRIMER v7.0.13 and IBMSPSS v.22. The patterns of species distribution between the four seasons were analyzed by shade plotting analysis from average standardized species abundance data. The species whose presence was available in all four seasons were defined as 'common species' in the community. The contribution of each species to the average Bray-Curtis similarity among the 14 trawls stations was calculated using SIMPER (similarity percentage analysis) program. Based on the SIMPER analysis, those 
species have a cumulative contribution of more than $70 \%$ in the total communities were defined as 'dominant species'. Both the dominant and common species identified as an important species in the fishery communities (Zhu and Tang, 2002; Shan et al., 2016). The significant differences of the fisheries community structure among the seasons were tested using ANOSIM (analysis of similarity) (Anderson et al., 2008; Clarke and Gorley, 2015). The seasonal variations in fishery assemblages were analyzed by coordination of the submodule of PCoA (Principal coordinates analysis) of PREMANOVA + of BrayCurtis similarities from square root transformed species abundance data (Anderson et al., 2008; Clarke and Gorley, 2015).

A simple Pearson correlation matrix was performed to identify an existing correlation among the dominant species of fishery communities using IBMSPSS v.22, and data were log-transformed before analysis.

\section{Results}

\section{Taxonomic composition and species distribution}

A total of 88 species belonging to 64 families were identified. These fishery samples included 43 fish species belonging to 28 families, 29 crustacean species belonging to 21 families, 12 mollusca species belonging to 11 families, three echinoderm species belonging to three families and one species of scyphozoan. The list of identified fishery species including their seasonal distribution, average abundance, frequency of occurrence was summarized in Table 2. Among the species, 21 species were reported common in terms of their presence in all four seasons, and eight species were found to be dominant with their higher contribution in the communities.

Based on the species distribution patterns, 19 species were present in three seasons, 18 species were present in two seasons, and 30 species were distributed only in one season (Fig. 2).

In terms of dominance (result from SIMPER analysis $>70 \%$ ), six species belonged to crustaceans (Alpheus japonicas, 15.34\%; Oratosquilla oratoria, 11.57\%; Palaemon gravieri, 10.57\%; Crangon affinis, 8.85\%; Acetes chinensis, $7.53 \%$; Leptochela gracilis, $7.19 \%$ ) and others were one fish species (Engraulis japonicas, 6.30\%) and one cephalopod species (Loligo japonica, 8.51\%) (Table 2 and Fig. 2).

In terms of species belonging to family, the maximum number of species were recorded under the family of Gobiidae with seven species (Amblychaeturichthys hexanema, Chaeturichthys stigmatias, Cryptocentrus filifer, Favonigobius gymnauchen, Ctenotrypauchen chinensis, Synechogobius hasta, and Odontamblyopus rubicundus), followed by the family of Engraulidae with five species (Coilia mystus, Engraulis japonicas, Setipinna taty, Thrissa kammalensis, and Thrissa mystax) and the Penaeidae with four species (Fenneropenaeus chinensis, Marsupenaeus japonicas, Metapenaeopsis dalei and Trachypenaeus curvirostris) (Table 2). 
Table 2. List of fishery species recorded in the Bohai Sea during a one-year cycle including their seasonal distribution, average abundance, frequency of occurrence and dominant species based on SIMPER analysis

\begin{tabular}{|c|c|c|c|c|c|c|c|c|c|c|c|}
\hline & \multirow{2}{*}{ Family } & \multirow{2}{*}{ Species name } & \multirow{2}{*}{ Dist. } & \multicolumn{2}{|c|}{ Winter } & \multicolumn{2}{|c|}{ Spring } & \multicolumn{2}{|c|}{ Summer } & \multicolumn{2}{|c|}{ Autumn } \\
\hline & & & & $N$ & $\%$ & $N$ & $\%$ & $N$ & $\%$ & $N$ & $\%$ \\
\hline \multirow{23}{*}{ Fish } & Ammodytidae & Ammodytes personatus (Girard, 1856) & ---+ & - & 0 & - & 100 & - & 0 & - & 0 \\
\hline & Apogonidae & Apogonichthys lineatus ((Temminck \& Schlegel, 1842) & --++ & - & 0 & - & 0 & + & 97.92 & - & 2.08 \\
\hline & Arcidae & Scapharca broughtonii (Schrenck, 1867) & -+-- & - & 0 & - & 100 & - & 0 & - & 0 \\
\hline & Callionymidae & Callionymus beniteguri (Jordan \& Snyder, 1900 & ++++ & + & 14.63 & - & 3.84 & + & 63.85 & + & 17.68 \\
\hline & Clupeidae & Konosirus punctatus ((Temminck \& Schlegel, 1846) & --+- & - & 0 & - & 0 & + & 100 & - & 0 \\
\hline & & Sardinella zunasi (Bleeker, 1854) & --+- & - & 0 & - & 0 & + & 100 & - & 0 \\
\hline & Cynoglossidae & Cynoglossus lighti (Norman, 1925) & ++++ & + & 20.85 & + & 24.24 & + & 18.43 & + & 36.48 \\
\hline & & Cynoglossus semilaevis (Günther, 1873) & +-+- & - & 46.67 & - & 0 & - & 53.33 & - & 0 \\
\hline & Engraulidae & Coilia mystus ((Linnaeus, 1758) & --++ & - & 0 & - & 100 & - & 0 & - & 0 \\
\hline & \multirow{10}{*}{ Gobiidae } & Engraulis japonicus (Temminck \& Schlegel, 1846) & -+++ & - & 0 & + & 1.32 & +++++ & 93.25 & +++ & 5.43 \\
\hline & & Thrissa kammalensis (Bleeker, 1849) & -+-- & - & 0 & + & 0 & + & 0 & + & 100 \\
\hline & & Thrissa mystax (Bloch \& Schneider, 1801) & -+++ & - & 0 & - & 9.62 & - & 82.52 & - & 7.86 \\
\hline & & Setipinna taty ((Valenciennes, 1848) & -++- & - & 1.61 & + & 81.92 & +++ & 15.38 & ++ & 1.08 \\
\hline & & Chaeturichthys stigmatias (Richardson, 1844) & ++++ & + & 6.91 & - & 0.18 & ++ & 57.36 & ++ & 35.55 \\
\hline & & Cryptocentrus filifer ((Valenciennes, 1837) & ++++ & - & 4.98 & + & 56.93 & - & 12.77 & - & 25.32 \\
\hline & & Ctenotrypauchen chinensis (Steindachner, 1867) & -++- & - & 0 & + & 11.95 & + & 60.51 & + & 27.54 \\
\hline & & Odontamblyopus rubicundus (Hamilton, 1822) & -+++ & - & 0 & - & 10.99 & - & 80.63 & - & 8.36 \\
\hline & & Favonigobius gymnauchen (Bleeker, 1860) & --+- & - & 0 & - & 0 & - & 100 & - & 0 \\
\hline & & Synechogobius hasta (Temminck \& Schlegel, 1845) & +-++ & + & 0 & - & 5.89 & - & 69.43 & - & 24.69 \\
\hline & Hexagrammidae & Hexagrammos otakii (Jordan \& Starks, 1895) & -+++ & - & 0 & + & 82.61 & - & 8.70 & - & 8.70 \\
\hline & Hemiramphidae & Hyporhamphus sajori ((Temminck \& Schlegel, 1846) & +--- & - & 100 & - & 0 & - & 0 & - & 0 \\
\hline & Liparidae & Liparis tanakae ((Gilbert \& Burke, 1912) & ++-+ & - & 0.06 & +++ & 99.87 & - & 0 & - & 0.07 \\
\hline & Lophiidae & Lophius litulon ((Jordan, 1902) & -+-- & - & 0 & - & 3.86 & + & 65.27 & + & 30.87 \\
\hline
\end{tabular}




\begin{tabular}{|c|c|c|c|c|c|c|c|c|c|c|c|}
\hline & \multirow{2}{*}{ Family } & \multirow{2}{*}{ Species name } & \multirow{2}{*}{ Dist. } & \multicolumn{2}{|c|}{ Winter } & \multicolumn{2}{|c|}{ Spring } & \multicolumn{2}{|c|}{ Summer } & \multicolumn{2}{|c|}{ Autumn } \\
\hline & & & & $N$ & $\%$ & $N$ & $\%$ & $N$ & $\%$ & $N$ & $\%$ \\
\hline & Monacanthidae & Navodon septentrionalis (Günther, 1874) & -+++ & - & 0 & - & 6.92 & - & 25.68 & - & 66.59 \\
\hline & Mugilidae & Liza haematocheila ((Temminck \& Schlegel, 1845) & +--- & - & 100 & - & 0 & - & 0 & - & 0 \\
\hline & Paralichthyidae & Paralichthys olivaceus ((Temminck \& Schlegel, 1846) & --+- & - & 0 & - & 99.97 & - & 0 & - & 0.03 \\
\hline & Pholidae & Enedrias fangi (Wang \& Wang, 1935) & +++- & - & 0.38 & +++ & 97.92 & + & 1.71 & - & 0 \\
\hline & Platycephalidae & Platycephalus indicus (Linnaeus, 1758) & -+-- & - & 0 & - & 2.30 & + & 56.21 & - & 41.49 \\
\hline & Pleuronectidae & Pseudopleuronectes yokohamae (Günther, 1877) & -+++ & - & 0 & - & 0 & - & 100 & - & 0 \\
\hline & Rajidae & Raja porosa (Günther, 1874) & --++ & - & 0 & - & 77.89 & - & 22.11 & - & 0 \\
\hline & Salangidae & Protosalanx chinensis (Osbeck, 1765) & ++++ & + & 50.13 & - & 100 & + & 0 & + & 0 \\
\hline & Sciaenidae & Argyrosomus argentatus (Houttuyn, 1782) & --+- & - & 0 & - & 0 & + & 100 & - & 0 \\
\hline & & Johnius belangerii (Cuvier, 1830) & -+-- & - & 0 & - & 35.48 & - & 64.52 & - & 0 \\
\hline & & Larimichthys polyactis ((Bleeker, 1877) & --++ & - & 0 & - & 0 & + & 99.51 & - & 0.49 \\
\hline & Scombridae & Scomber japonicus (Houttuyn, 1782) & --+- & - & 0 & - & 0 & + & 100 & - & 0 \\
\hline & & Scomberomorrus niphonius (Cuvier, 1832) & --+- & - & 0 & - & 0 & + & 100 & - & 0 \\
\hline & Sebastidae & Sebastes schlegeli (Hilgendorf, 1880) & ++++ & - & 0.67 & + & 6.16 & + & 92.78 & - & 0.39 \\
\hline & Stromateidae & Pampus argenteus (Euphrasen, 1788) & --++ & - & 0 & - & 100 & - & 0 & - & 0 \\
\hline & Syngnathidae & Syngnathus acus (Linnaeus, 1758) & -+++ & - & 65.32 & - & 0 & - & 5.99 & - & 28.69 \\
\hline & Tetraodontidae & Takifugu vermicularis (Temminck \& Schlegel, 1850) & ---+ & - & 0 & - & 0 & - & 90 & - & 10 \\
\hline & Trichiuridae & Eupleurogrammus muticus (Gray, 1831) & -+-- & - & 0 & - & 13.90 & + & 56.95 & + & 29.15 \\
\hline & Zoarcidae & Zoarces elongates (Kner, 1868) & -+++ & - & 0 & - & 100 & - & 0 & - & 0 \\
\hline \multirow{8}{*}{ Crustacean } & Alpheidae & Alpheus distinguendus (de Man, 1909) & --++ & - & 0 & - & 0 & + & 6.16 & + & 93.84 \\
\hline & & Alpheus heterocarpus (Yu, 1935) & ++-- & - & 7.99 & + & 92.01 & - & 0 & - & 0 \\
\hline & & Alpheus japonicus (Miers, 1879) & ++++ & +++ & 32.62 & + & 3.89 & ++ & 16.83 & +++ & 46.65 \\
\hline & Cancridae & Cancer gibbosulus (De Haan, 1833) & -++- & - & 0 & + & 75.51 & - & 24.49 & - & 0 \\
\hline & Crangonidae & Crangon affinis (De Haan, 1849) & ++++ & + & 6.04 & ++++ & 85.18 & + & 0.36 & + & 8.43 \\
\hline & Dorippidae & Dorippe japonica (von Seibold, 1824) & +++- & - & 0.62 & - & 2.13 & + & 97.25 & - & 0 \\
\hline & Euryplacidae & Eucrate crenata (De Haan, 1835) & --++ & - & 0 & - & 0 & + & 99.11 & - & 0.89 \\
\hline & Euphausiidae & Euphausia pacifica (Hansen, 1911) & -+++ & - & 0 & - & 0 & - & 0 & - & 0 \\
\hline
\end{tabular}




\begin{tabular}{|c|c|c|c|c|c|c|c|c|c|c|c|}
\hline & \multirow{2}{*}{ Family } & \multirow{2}{*}{ Species name } & \multirow{2}{*}{ Dist. } & \multicolumn{2}{|c|}{ Winter } & \multicolumn{2}{|c|}{ Spring } & \multicolumn{2}{|c|}{ Summer } & \multicolumn{2}{|c|}{ Autumn } \\
\hline & & & & $N$ & $\%$ & $N$ & $\%$ & $N$ & $\%$ & $N$ & $\%$ \\
\hline & Goneplacidae & Carcinoplax vestita (De Haan, 1835) & ++++ & + & 42.07 & + & 12.38 & + & 31.32 & + & 14.22 \\
\hline & Hemiramphidae & Hyporhamphus limbatus (Valenciennes, 1847) & +--- & - & 100 & - & 0 & - & 0 & - & 0 \\
\hline & Hippolytidae & Latreutes anoplonyx (Kemp, 1914) & ++-- & + & 71.79 & + & 28.21 & - & 0 & - & 0 \\
\hline & & Latreutes planirostris (De Haan, 1844) & +-++ & - & 0.42 & - & 0 & + & 92.29 & + & 7.28 \\
\hline & Paguridae & Paguridae (Latreille, 1802) & -+-+ & - & 0 & + & 21.23 & + & 5.89 & - & 26.84 \\
\hline & Leucosiidae & Arcania heptacantha (De Man, 1907) & --+- & - & 0 & - & 0 & - & 100 & - & 0 \\
\hline & Palaemonidae & Palaemon gravieri $((\mathrm{Yu}, 1930)$ & ++++ & ++ & 46.06 & + & 0 & + & 62.12 & + & 37.88 \\
\hline & Pasiphaeidae & Leptochela gracilis (Stimpson, 1860) & -+++ & - & 0 & + & 13.10 & + & 0.35 & ++++ & 86.55 \\
\hline & Portunidae & Charybdis bimaculata (Miers, 1886) & ++++ & - & 1.32 & - & 10.52 & + & 23.85 & + & 64.32 \\
\hline & & Charybdis japonica (A. Milne-Edwards, 1861) & ++++ & - & 0.40 & - & 4.62 & + & 94.51 & - & 0.46 \\
\hline & & Portunus trituberculatus (Miers, 1876) & -+++ & - & 0 & - & 4.23 & + & 22.07 & + & 23.57 \\
\hline & Penaeidae & Fenneropenaeus chinensis (Osbeck, 1765) & --+- & - & 0 & - & 0 & + & 100 & - & 0 \\
\hline & & Marsupenaeus japonicus (Spence Bate, 1888) & --+- & - & 0 & - & 0 & - & 100 & - & 0 \\
\hline & & Metapenaeopsis dalei ((Rathbun, 1902) & -+++ & - & 0 & - & 9.27 & - & 0 & + & 90.73 \\
\hline & & Trachypenaeus curvirostris (Balss, 1933) & -++- & - & 0 & - & 0 & + & 0 & + & 100 \\
\hline & Pinnotheridae & Pinnotheridae (De Haan, 1833) & ---+ & - & 0 & - & 10.43 & - & 89.57 & + & 0 \\
\hline & Macrophthalmidae & Tritodynamia rathbunae (Shen, 1932) & --++ & - & 0 & - & 0.75 & + & 7.55 & - & 91.70 \\
\hline & Matutidae & Matuta planipes (Fabricius, 1798) & --+- & - & 0 & - & 0 & + & 100 & - & 0 \\
\hline & Sergestidae & Acetes chinensis (Hansen, 199) & ++++ & +++ & 62.64 & + & 7.28 & + & 15.73 & + & 14.35 \\
\hline & Squillidae & Oratosquilla oratoria (De Haan, 1844) & ++++ & - & 0.03 & +++ & 60.08 & +++++ & 39.92 & ++ & 0 \\
\hline & Upogebiidae & Austinogebia edulis (Ngoc-Ho \& Chan, 1992) & ---- & - & 0 & - & 0.54 & - & 0.54 & + & 98.92 \\
\hline \multirow{6}{*}{ Mollusca } & Hiatellidae & 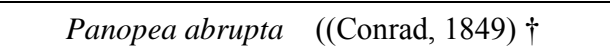 & \begin{tabular}{c|c}
-+- \\
-+
\end{tabular} & - & 0 & - & 21.69 & - & 42.15 & - & 36.16 \\
\hline & Loliginidae & Loligo japonica (Hoyle, 1885) & ++++ & - & 0.17 & + & 6.40 & ++++ & 76.82 & ++ & 16.61 \\
\hline & Muricidae & Rapana venosa ((Valenciennes, 1846) & -+-- & - & 0 & + & 0 & - & 100 & - & 0 \\
\hline & Mytilidae & Mytilus edulis (Linnaeus, 1758) & +-+ & - & 0 & - & 0 & - & 100 & - & 0 \\
\hline & Nassariidae & Nassariidae (Iredale, 1916 (1835)) & --+- & - & 0 & - & 100 & - & 0 & - & 0 \\
\hline & Naticidae & Glossaulax didyma ((Röding, 1798) & ++++ & - & 5.74 & + & 88.52 & - & 4.92 & - & 0.82 \\
\hline
\end{tabular}




\begin{tabular}{|c|c|c|c|c|c|c|c|c|c|c|c|}
\hline & \multirow{2}{*}{ Family } & \multirow{2}{*}{ Species name } & \multirow{2}{*}{ Dist. } & \multicolumn{2}{|c|}{ Winter } & \multicolumn{2}{|c|}{ Spring } & \multicolumn{2}{|c|}{ Summer } & \multicolumn{2}{|c|}{ Autumn } \\
\hline & & & & $N$ & $\%$ & $N$ & $\%$ & $N$ & $\%$ & $N$ & $\%$ \\
\hline & Nautilidae & Nautiloidea sp. & -+-- & - & 0 & - & 0 & - & 50 & - & 50 \\
\hline & Octopodidae & Octopus ocellatus (Gray, 1849) & ++++ & - & 0.81 & + & 45.61 & + & 34.20 & + & 15.20 \\
\hline & & Octopus variablilis ((Sasaki, 1929) & ++++ & - & 4.99 & - & 0 & - & 100 & - & 0 \\
\hline & Philinidae & Philine kinglipini (Tchang, 1934) & -+-+ & - & 0 & ++++ & 100 & - & 0 & - & 0 \\
\hline & Pinnidae & Pinna rudis (Linnaeus, 1758) & --+- & - & 0 & - & 0 & - & 0 & - & 100 \\
\hline & Sepiolidae & Sepiola birostrata (Sasaki, 1918) & ++++ & - & 0 & + & 0 & + & 0 & - & 0 \\
\hline \multirow{4}{*}{ Echinod. } & Asteriidae & Asierias rollestoni (Bell, 1881) & -+++ & - & 0 & + & 44.06 & - & 11.59 & + & 44.35 \\
\hline & Luidiidae & Luidia yesoensis (Goto, 1914) & ++-+ & + & 18.25 & + & 68.45 & - & 0 & + & 13.30 \\
\hline & Strongylocentrotidae & Hemicentrotus pulcherrimus (A. Agassiz, 1864) & -+-- & + & 25.37 & + & 6.06 & - & 0.38 & + & 68.19 \\
\hline & Scyphozoa (Class) & Scyphozoa sp. & ++++ & + & 98 & - & 0 & - & 2 & - & 0 \\
\hline
\end{tabular}

Text bold, dominant species; Dist., distribution: +, present; -, absent; $N$, average abundance: > 500,"+++++", > 200,"++++", > 100,"+++", > 50,"++", > 1,"+","-"; \%, frequency of occurrence 
According to the frequency of occurrence of the dominant species, Acetes chinensis, Palaemon gravieri, and Alpheus japonicas were accounting for $62.64 \%, 46.06 \%$, and $32.62 \%$, respectively in the winter season (January) while Crangon affinis and Oratosquilla oratoria were accounting for $85.18 \%$ and $60.08 \%$, respectively in the spring season (May). In the summer season (August) Engraulis japonicus, Loligo japonica, Palaemon gravieri, and Oratosquilla oratoria were accounting for 93.25\%, 76.82\%, $62.12 \%$, and 39.92\%, respectively whereas Alpheus japonicas, Leptochela gracilis and Palaemon gravieri were accounting for $46.65 \%, 86.55 \%, 37.88 \%$, respectively in the autumn season (November) (Table 2 and Fig. 2).

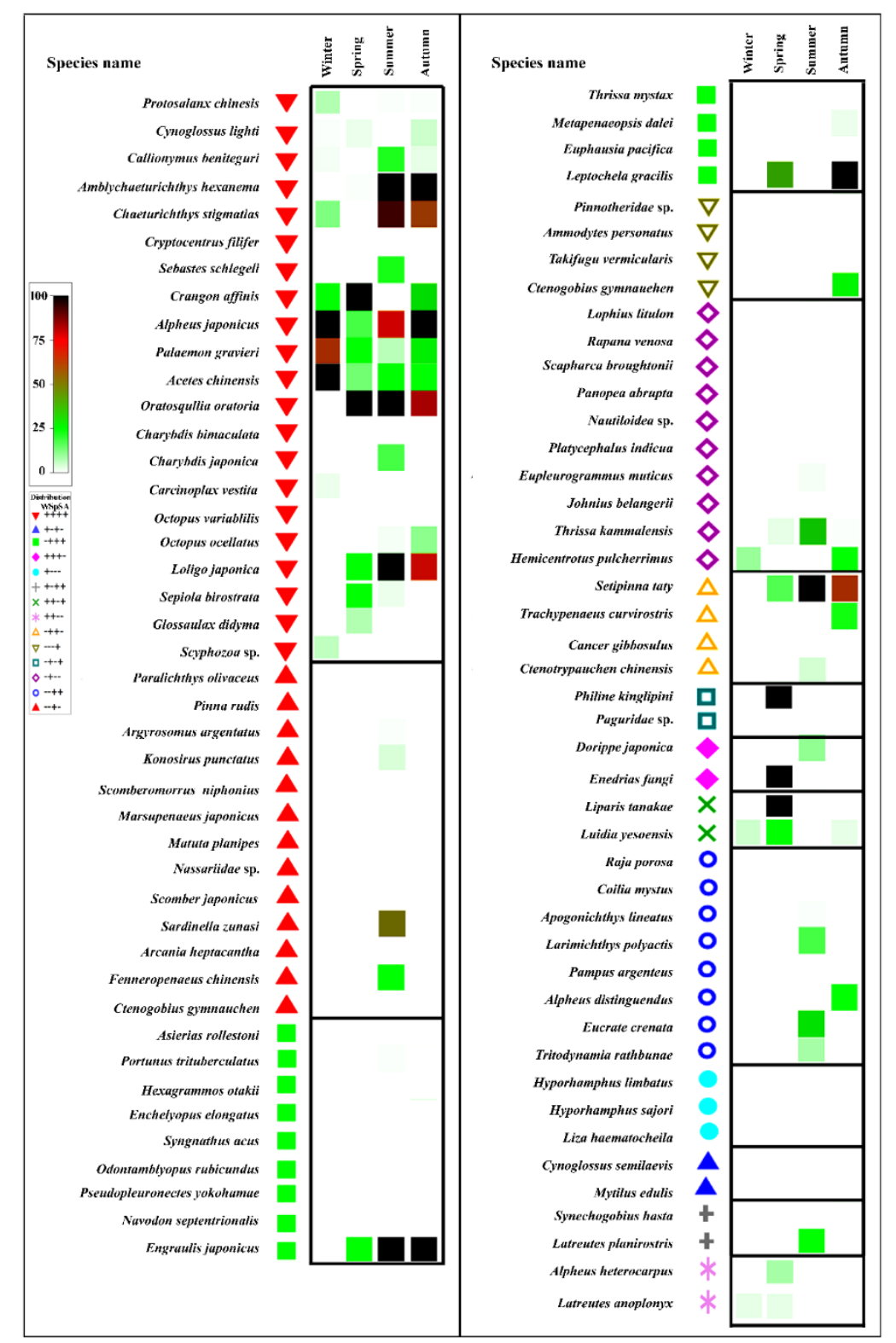

Figure 2. Shade plot showing the seasonal distribution pattern of fishery species in terms of their relative abundance during the study period in the Bohai Sea 


\section{Seasonal variation in fishery dynamics}

In terms of abundance of dominant species per haul, the individual abundance found to be higher in the summer and lower in the winter (Fig. 3a). As to relative abundance, three species of crustaceans were predominant in the winter (Alpheus japonicas, Acetes chinensis and Palaemon gravieri); two species in the spring (Crangos affinis and Oratosquilla oratoria); two species in the summer (Engraulis japonicus and Oratosquilla oratoria); and two species in the autumn season (Leptochela gracilis and Alpheus japonicus) (Fig. 3b).

Based on species occurrence, the maximum species occurred in summer and autumn, whereas the minimum was in winter (Fig. 4a). For example, of these 88 species, 24 were occurred in summer and autumn, while only seven species were in winter. In terms of total abundance, the highest abundance recorded in summer, while the lowest was in winter (Fig. 4b).

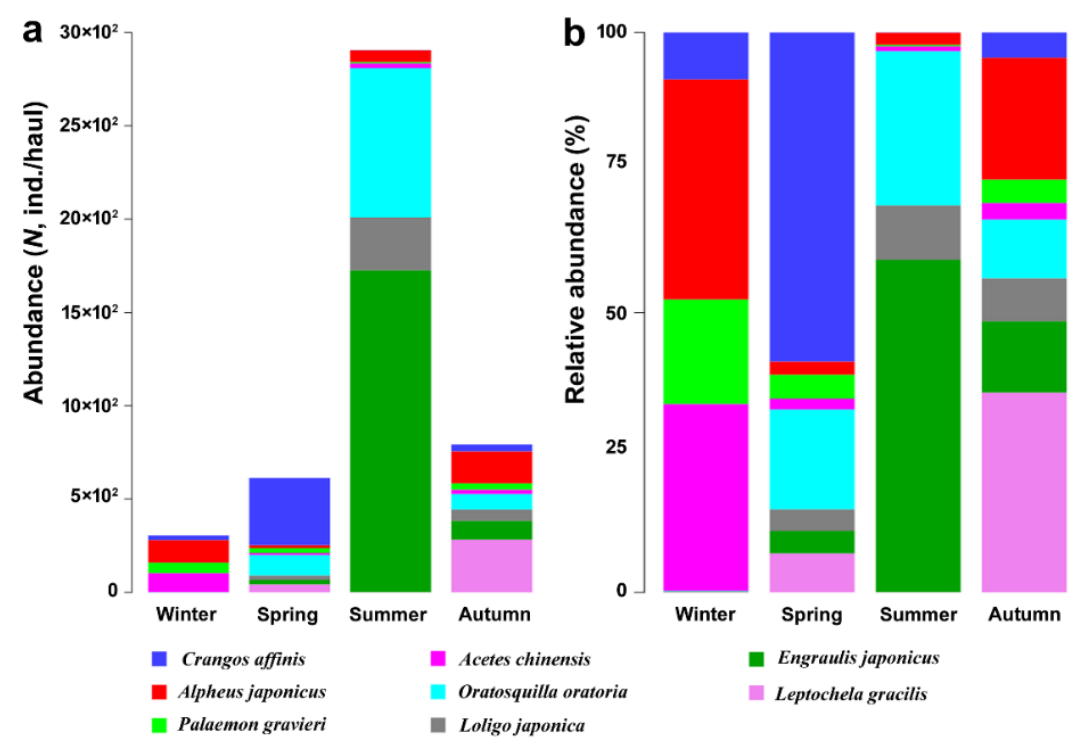

Figure 3. Variation in abundance and relative abundance of dominant species of fishery species during the study period in the Bohai Sea

The analysis of similarity (ANOSIM) revealed that there was a significant difference in fishery community structure between the four seasons (Global, $\mathrm{R}=0.467, P<0.001$ ). Based on SIMPER analysis, seven typical species (Crangon affinis, Enedrias fangi, Oratosquilla oratoria, Acetes chinensis, Alpheus japonicas, Liparis tanakae and Philine kinglipini) driven 93.34\% average dissimilarities between winter and spring seasons. Similarly, six species (Engraulis japonicas, Oratosquilla oratoria, Loligo japonica, Alpheus japonicas, Acetes chinensis and Chaeturichthys stigmatias) in winter and spring seasons (97.33\%); eight species (Leptochela gracilis, Alpheus japonicas, Acetes chinensis, Amblychaeturichthys hexanema, Engraulis japonicas, Setipinna taty, Palaemon gravieri, and Crangon affinis) in winter and autumn seasons (90.37\%); seven species (Engraulis japonicas, Oratosquilla oratoria, Loligo japonica, Crangon affinis, Amblychaeturichthys hexanema, Setipinna taty and Enedrias fangi) in spring and summer seasons (95.07); nine species (Leptochela gracilis, Crangon affinis, 
Enedrias fangi, Amblychaeturichthys hexanema, Oratosquilla oratoria, Liparis tanakae, Philine kinglipini, Alpheus japonicas and Engraulis japonicus) in spring and autumn seasons (89.81\%); and six species (Engraulis japonicas, Oratosquilla oratoria, Loligo japonica, Amblychaeturichthys hexanema, Leptochela gracilis, and Setipinna taty) in summer and autumn seasons (92.29\%) (Table 3).
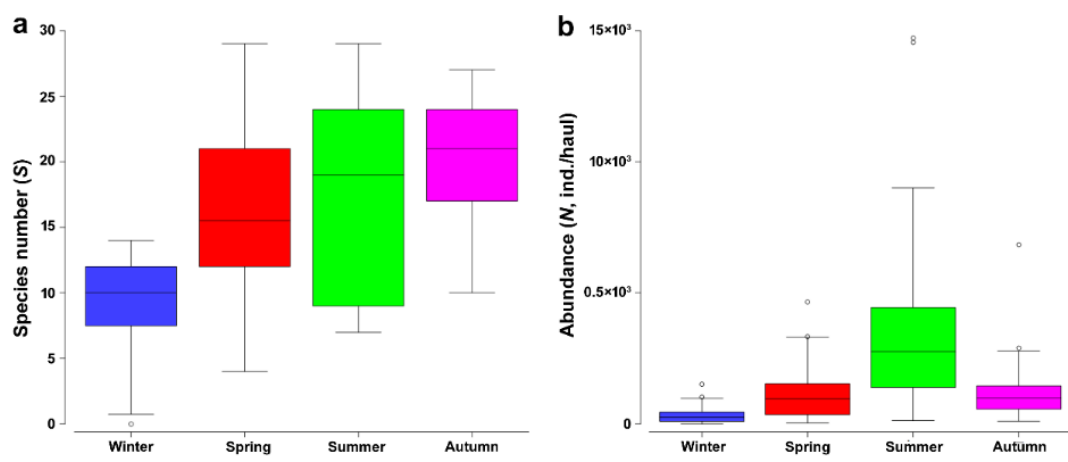

Figure 4. Seasonal variation in species number (a) and total abundance (b) in the fishery species during the study period in the Bohai Sea

\section{Seasonal variation in diversity pattern of fishery species}

Among the community parameters, the species richness showed increasing trend from winter to autumn season, with a higher value in the autumn and lower in winter while species evenness showed opposite that decreased from winter to summer, then again increased in autumn season with a value higher in winter and lower in summer season (Fig. 5a,b). The species diversity $\left(\mathrm{H}^{\prime}\right)$ and Simpson index $\left(1-\lambda^{\prime}\right)$ showed similar seasonal patterns and both indices values were higher in autumn and lower in the summer season (Fig. $5 c, d)$.

In terms of fishery diversity status, there was a clear temporal variation revealed in the Bohai Sea during the study period (Fig. 5). For example, the highest value of species richness (3.79) was found in the autumn season whereas it was the lowest (1.65) in the winter with an average of $2.80 \pm 0.59$. The species evenness was found to be maximum (0.95) in the winter season and minimum (0.18) in the summer season with an average of $0.63 \pm 0.17$. The maximum species diversity index was (2.31) recorded in autumn season and minimum (0.57) was in summer season with an average of $1.82 \pm 0.44$ while Simpson index was found to be similar with diversity that was the highest $(0.88)$ in autumn season and the lowest $(0.18)$ in summer season with an average of $0.73 \pm 0.18$ (Fig. 5).

\section{Patterns of community structural of fishery species}

Principal analysis of coordinates (PCoA) summarized the seasonal structural variations in the fishery species. The first canonical axis (PCoA1) separated the samples in winter and spring (on the left) from those in summer and autumn (on the right), while the second canonical axis (PCoA2) discriminated the samples in winter and summer (upper) from those at the others two seasons spring and autumn (lower) (Fig. 6a). 
Table 3. Results of the one-way analysis of similarity (ANOSIM) showing the significant differences and SIMPER results of the typical species driven the seasonal variation in the fishery species during the study period in the Bohai Sea

\begin{tabular}{|c|c|c|c|c|c|}
\hline \multirow{2}{*}{$\begin{array}{c}\text { Global } \\
R=0.467, P=0.001\end{array}$} & \multicolumn{2}{|c|}{ ANOSIM } & \multicolumn{2}{|c|}{ Dissimilarity index from SIMPER } & \multirow{2}{*}{ Cont. $(\%)$} \\
\hline & $\mathbf{R}$ & $\mathbf{P}$ & Ave. Diss. (\%) & Typical species & \\
\hline $\begin{array}{l}\text { Winter vs } \\
\text { Spring }\end{array}$ & 0.531 & 0.1 & 93.34 & $\begin{array}{l}\text { Crangon affinis } \\
\text { Enedrias fangi } \\
\text { Oratosquilla oratoria } \\
\text { Acetes chinensis } \\
\text { Alpheus japonicas } \\
\text { Liparis tanakae } \\
\text { Philine kinglipini } \\
\end{array}$ & $\begin{array}{l}17.28 \\
10.02 \\
9.95 \\
9.57 \\
8.98 \\
8.57 \\
6.07\end{array}$ \\
\hline $\begin{array}{l}\text { Winter vs } \\
\text { Summer }\end{array}$ & 0.593 & 0.1 & 97.33 & $\begin{array}{c}\text { Engraulis japonicas } \\
\text { Oratosquilla oratoria } \\
\text { Loligo japonica } \\
\text { Alpheus japonicas } \\
\text { Acetes chinensis } \\
\text { Chaeturichthys stigmatias }\end{array}$ & $\begin{array}{c}27.82 \\
18.46 \\
12.25 \\
5.17 \\
5.09 \\
5.04\end{array}$ \\
\hline $\begin{array}{l}\text { Winter vs } \\
\text { Autumn }\end{array}$ & 0.485 & 0.1 & 90.37 & $\begin{array}{c}\text { Leptochela gracilis } \\
\text { Alpheus japonicas } \\
\text { Acetes chinensis } \\
\text { Amblychaeturichthys hexanema } \\
\text { Engraulis japonicas } \\
\text { Setipinna taty } \\
\text { Palaemon gravieri } \\
\text { Crangon affinis }\end{array}$ & $\begin{array}{l}19.71 \\
11.31 \\
9.00 \\
8.55 \\
6.30 \\
5.78 \\
5.45 \\
4.81 \\
\end{array}$ \\
\hline $\begin{array}{l}\text { Spring vs } \\
\text { Summer }\end{array}$ & 0.449 & 0.1 & 95.07 & $\begin{array}{c}\text { Engraulis japonicas } \\
\text { Oratosquilla oratoria } \\
\text { Loligo japonica } \\
\text { Crangon affinis } \\
\text { Amblychaeturichthys hexanema } \\
\text { Setipinna taty } \\
\text { Enedrias fangi }\end{array}$ & $\begin{array}{l}23.82 \\
17.22 \\
9.56 \\
8.22 \\
4.84 \\
4.69 \\
4.65\end{array}$ \\
\hline $\begin{array}{l}\text { Spring vs } \\
\text { Autumn }\end{array}$ & 0.369 & 0.1 & 89.81 & $\begin{array}{c}\text { Leptochela gracilis } \\
\text { Crangon affinis } \\
\text { Enedrias fangi } \\
\text { Amblychaeturichthys hexanema } \\
\text { Oratosquilla oratoria } \\
\text { Liparis tanakae } \\
\text { Philine kinglipini } \\
\text { Alpheus japonicas } \\
\text { Engraulis japonicus } \\
\end{array}$ & $\begin{array}{l}15.11 \\
13.55 \\
7.47 \\
7.01 \\
6.87 \\
5.84 \\
5.46 \\
5.41 \\
5.23 \\
\end{array}$ \\
\hline $\begin{array}{l}\text { Summer vs } \\
\text { Autumn }\end{array}$ & 0.359 & 0.1 & 92.29 & $\begin{array}{c}\text { Engraulis japonicas } \\
\text { Oratosquilla oratoria } \\
\text { Loligo japonica } \\
\text { Amblychaeturichthys hexanema } \\
\text { Leptochela gracilis } \\
\text { Setipinna taty } \\
\end{array}$ & $\begin{array}{l}24.26 \\
16.48 \\
9.74 \\
9.52 \\
9.02 \\
6.27\end{array}$ \\
\hline
\end{tabular}

Ave. Diss., average dissimilarity; Cont., contribution

Vector overlay of Pearson correlations of eight dominant species revealed that vectors of four species (Engraulis japonicas, Oratosquilla oratoria, Loligo japonica and Leptochela gracilis) pointed towards the sample clouds in summer season (upper right), three species (Alpheus japonicas, Acetes chinensis and Palaemon gravieri) of crustacean towards that in winter season (upper left), and one species (Crangos affinis) toward in spring season (lower left) (Fig. 6b). 
A simple Pearson correlation among the eight dominant species was summarized in Table 4 showed a significant correlation during the study period. Among these eight species, only four species showed significant correlations. For example, Palaemon gravieri showed a positively significant correlation with Alpheus japonicus ( $\mathrm{r}=0.427^{* *}$, $\mathrm{P}<0.01)$ whereas Loligo japonica correlated with Oratosquilla oratoria $\left(\mathrm{r}=0.517^{* *}\right.$, $\mathrm{P}<0.01)$ (Table 4).
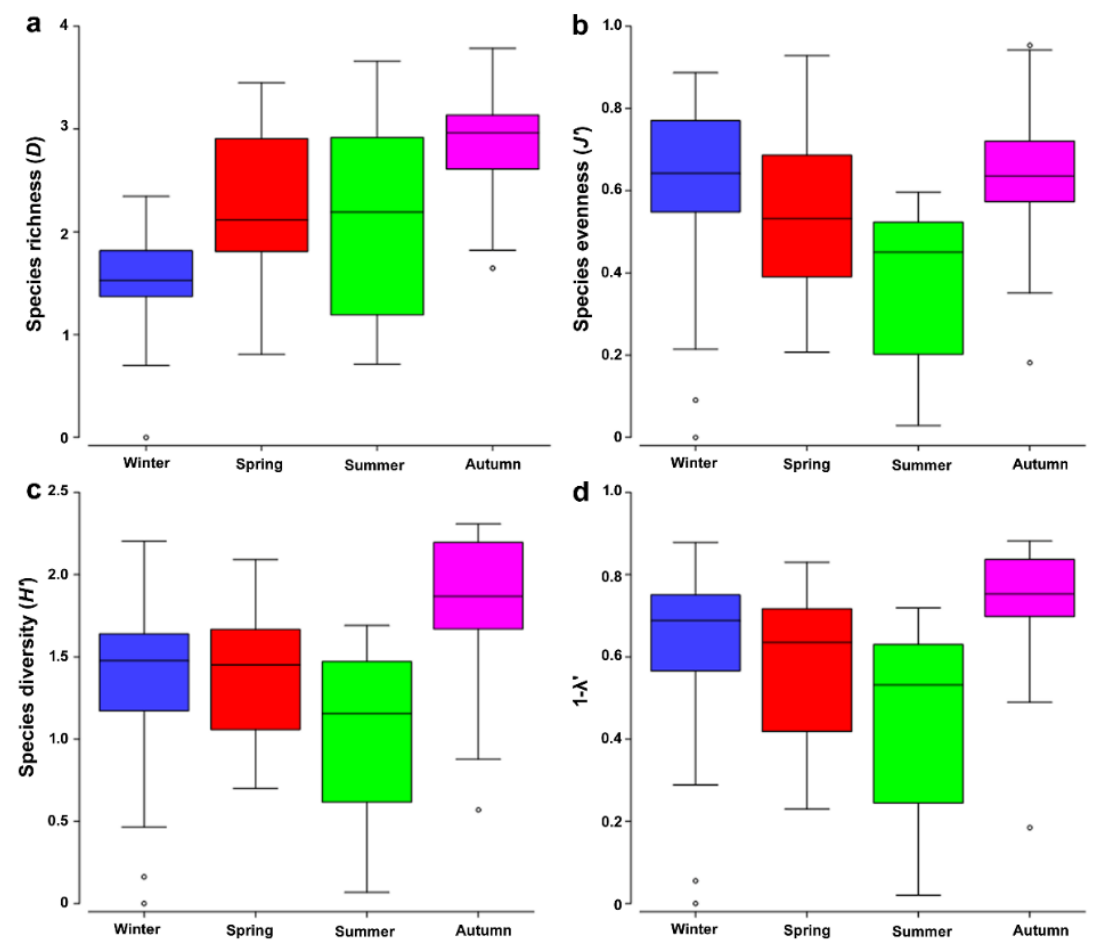

Figure 5. Variation in species richness (a), evenness (b), diversity (c) and Simpson index (d) during the study period in the Bohai Sea [Whiskers, minimum and maximum; boxes, $\pm 25 \%$; lines, medians]
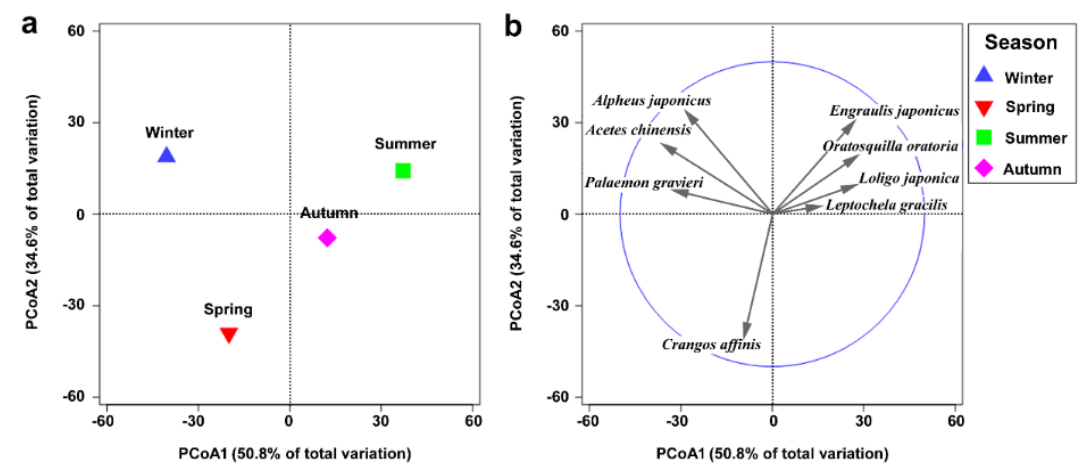

Figure 6. Principle coordinate analysis ( $P C O A)$ on Bray-Curtis similarities from square root transformed species abundance data (a); with vector correlation of eight dominant species with the PCoA axis (b), showing their seasonal variation in community structure of fishery species 
Table 4. Pearson correlation coefficients among the eight dominant species during the study period in the Bohai Sea

\begin{tabular}{|c|c|c|c|c|c|c|c|c|}
\hline & $\begin{array}{c}\text { Crangos } \\
\text { affinis }\end{array}$ & $\begin{array}{l}\text { Alpheus } \\
\text { japonicus }\end{array}$ & $\begin{array}{c}\text { Palaemon } \\
\text { gravieri }\end{array}$ & $\begin{array}{c}\text { Acetes } \\
\text { chinensis }\end{array}$ & $\begin{array}{c}\text { Oratosquilla } \\
\text { oratoria }\end{array}$ & $\begin{array}{c}\text { Loligo } \\
\text { japonica }\end{array}$ & $\begin{array}{l}\text { Engraulis } \\
\text { japonicus }\end{array}$ & $\begin{array}{c}\text { Leptochela } \\
\text { gracilis }\end{array}$ \\
\hline Crangos affinis & 1 & -.090 & .050 & -.066 & -.053 & -.115 & -.056 & .005 \\
\hline $\begin{array}{l}\text { Alpheus } \\
\text { japonicus }\end{array}$ & & 1 & $.427 * *$ & -.073 & .117 & .142 & -.064 & -.065 \\
\hline $\begin{array}{c}\text { Palaemon } \\
\text { gravieri }\end{array}$ & & & 1 & -.040 & -.110 & -.149 & -.119 & .058 \\
\hline Acetes chinensis & & & & 1 & -.031 & -.040 & -.067 & -.058 \\
\hline $\begin{array}{c}\text { Oratosquilla } \\
\text { oratoria }\end{array}$ & & & & & 1 & $.517 * *$ & -.075 & -.110 \\
\hline Loligo japonica & & & & & & 1 & -.061 & -.129 \\
\hline $\begin{array}{l}\text { Engraulis } \\
\text { japonicus }\end{array}$ & & & & & & & 1 & -.068 \\
\hline $\begin{array}{c}\text { Leptochela } \\
\text { gracilis }\end{array}$ & & & & & & & & 1 \\
\hline
\end{tabular}

Text bold, significant values; $* *$, significant level at $0.05(P<0.01)$

\section{Discussion}

The Bohai Sea is considered as an important fishery ground (i.e., spawning, nursing, and fishing) in northern China and the fishery resources have a dynamic nature (Chen et al., 1997; Deng and Jin, 2001). For instance, this shallow water mass used as an important migratory route for many marine species from the Yellow Sea (Liu, 1990). However, the increasing pressure of anthropogenic activities and climate variabilities have already altered the Bohai Sea ecosystem (Lin et al., 2001; Ning et al., 2010; Guo et al., 2013; Liu and Zhang, 2013; Pelling et al., 2013). Investigations have documented that change in climatic conditions and overfishing are the main drivers for fast-paced structural change in the marine ecosystem (Planque et al., 2010; Aschan et al., 2013; Rocha et al., 2015). In this study, we evaluated the characteristics of community structure and dynamics of fishery species in the Bohai Sea.

The Bohai Sea ecosystem is mainly composed of warm water and warm-temperate species. Previous research based on the ecotypes features (i.e., warm-water, warmtemperate, and cold-temperate species), a total of 97 fish species were reported over six decades where a rapid shifting of dominant species occurred (Shan et al., 2016). After 1982, both in the spring and summer season, the species of that ecotype decreased. In an earlier study in the Yellow River estuary of Laizhou Bay in the Bohai Sea, reported that 77 fish species were accounted for where eight pelagic fishes catch contributed more than $10 \%$ in every sampling year (Shan et al., 2013). The species composition changed during the survey periods in the Yellow River estuary. Another survey results in the Yellow and 
Bohai Seas, reported a total of 126 species comprising 102 fishes, 17 crustaceans, five cephalopods and two medusas where Acetes chinensis was the most dominant in terms of biomass (Chen et al., 1997). Thirty-two crustacean species were enlisted, among them only single species Crangon affinis was the dominant species both in Yellow and Bohai Seas during the spring season (Wu et al., 2012). In our study results, Crangon affinis was found to be the dominant species which is consisted of previous research results. In addition, our present study found that major dominant species were belonging to crustacean group (Alpheus japonicas, 15.34\%; Oratosquilla oratoria, 11.57\%; Palaemon gravieri, 10.57\%; Crangon affinis, 8.85\%; Acetes chinensis, 7.53\%; Leptochela gracilis, $7.19 \%$ ) which clearly indicated the dominant fishery species shift in this ecosystem. The small-sized, pelagic fishes (Engraulis japonicas, 6.30\%), and crustaceans' species prevailed in the Bohai Sea ecosystem were also reported (Xu and Jin, 2005; Wang et al., 2010; Li et al., 2013).

Based on species richness and total abundance, the maximum was recorded in the summer season. In addition, abundance of eight dominant species also showed similar community variation, thus might be due to variation in ecological conditions among the seasons. Several studies have stated that environmental factors (e.g., temperature, transparency, salinity, dissolved oxygen, nutrients, and water $\mathrm{pH}$ ) can significantly influence the ecological condition of water in both temporal and spatial scales. Therefore, this ecological condition may shape the fishery species in the Bohai Sea ecosystem (Lin et al., 2001; Jin et al., 2013; Shan et al., 2016). The present study found that there was a significant seasonal shift of dominant fishery species which might be due to fluctuation of water parameters (e.g., temperature) over the decades (Jin 2004; Shan et al., 2016). Many studies showed that recent climate-change-induced variabilities negatively affected the pelagic fishery productivity (Jin et al., 2003; Stige et al., 2006; Perry et al., 2010). The changes in variabilities impede the supply of primary productivity and breakdown the interaction of the trophic levels. So the resultant of this lower food supply can affect the fishery recruitment patterns and dynamics by limiting species migration and shifting keystone species (Zhu and Tang, 2002; Ning et al., 2010) (Table 5).

Diversity indices are widely used in the representation of the homogeneity and heterogeneity of ecosystem status, such as existing fishery status on species levels (Shan et al., 2010a, 2011, 2013; Chen et al., 2018). Generally, higher indices values indicate a better environment/ecological quality status and represent a stable ecosystem condition (Chen et al., 1997; Shan et al., 2010b). In the present study we found, species richness, Simpson indices, and diversity were higher in the autumn season which indicated that the ecological condition of water was relatively favorable for fishery species in this season compared to the other three seasons. This variation might be occurred due to the fluctuation of water temperature in the Bohai Sea (Xia and Xiong, 2013). Besides, several anthropogenic stresses (e.g., both municipal/land-based pollution, beerier of natural water flow, coastal reclamation activities) have significant negative effects on fishery species composition and distribution (Zhao and Kong, 2000; Cui et al., 2005; Shan et al., 2013; Yan et al., 2013). In addition, changing of the ecological condition due to algal blooms in winter have a significant negative impact on fishery species was reported in elsewhere 
which is consistent with the present study findings that fishery stock turns over in autumn season just after winter season (Wang et al., 2006; Tang et al., 2010). Thus, the findings of the present study suggest that the community patterns and dynamics structure of fishery species have closed interactions with climate and water ecological conditions in the Bohai Sea.

Multivariate analysis is a more useful tool than univariate analysis for summarizing the interaction of biotic communities with abiotic factors (Anderson et al., 2008; Clark and Gorley, 2015; Chen et al., 2018). Based on these strategies in the present study, PCoA (principal coordinates analysis) and ANOSIM (analysis of similarity) demonstrated that there was an apparent significant seasonal variation in community patterns and dynamics of fishery species in the Bohai Sea ecosystem. This variation is mainly driven by eight dominant species and their mutual interactions (Table 4) with typical species (Table 3).

Table 5. Fishery species composition in different studies in the Northwest Pacific Ocean

\begin{tabular}{|c|c|c|c|c|c|c|}
\hline Location & Fishes & Crustaceans & Molluses & Echinod. & Scyphozoan & References \\
\hline Bohai Sea & 43 & 29 & 12 & 3 & 1 & Present study \\
\hline Bohai Sea & 97 & - & - & - & - & Shan et al., 2016 \\
\hline Bohai Sea & 75 & 10 & 6 & - & - & Jin, 2004 \\
\hline Yellow River & & & & & & \\
\hline $\begin{array}{c}\text { estuary ecosystem } \\
\text { in the southern } \\
\text { Bohai Sea }\end{array}$ & 77 & - & - & - & - & Shan et al., 2013 \\
\hline $\begin{array}{c}\text { Yellow and Bohai } \\
\text { Sea }\end{array}$ & 102 & 17 & 5 & - & 2 & $\begin{array}{l}\text { Chen et al., } \\
1997\end{array}$ \\
\hline Yellow Sea & $\begin{array}{c}214 \text { (in 1959), } 351 \\
\text { (in 1985) }\end{array}$ & - & - & - & - & $\begin{array}{c}\text { Jin and Tang } \\
1996\end{array}$ \\
\hline $\begin{array}{l}\text { Middle Yellow } \\
\text { Sea of Korea }\end{array}$ & 40 & 22 & 17 & 2 & - & Lee et al., 2010 \\
\hline $\begin{array}{l}\text { East China Sea } \\
\text { and southern } \\
\text { Yellow Sea }\end{array}$ & $\begin{array}{l}149 \text { (in autumn), } \\
177 \text { (in spring) }\end{array}$ & - & - & - & - & Jin et al., 2003 \\
\hline $\begin{array}{c}\text { Middle continental } \\
\text { shelf of the East } \\
\text { China Sea }\end{array}$ & 186 & - & - & - & - & Shan et al., 2011 \\
\hline
\end{tabular}

\section{Conclusion}

The shallow Bohai Sea ecosystem is used as a unique ground for spawning, feeding, and nursery for many commercial as well as ecological fishery species. The structural patterns and community dynamics of the fishery species in the Bohai Sea are complex and have complicated linked with multiple factors (e.g., pollution, overfishing, climate change, and water ecology). In our present study used a pair trawl survey data to observe the seasonal variation in community patterns and structural dynamics fishery species especially, their abundance, composition, distribution and dominant shift in the Bohai Sea. The community parameters mainly, species richness, diversity, and Simpson indices were higher in the autumn. Multivariate analyses revealed a significant seasonal variation in the fishery community structure patterns and structural dynamics. Our results suggest that ecological conditions may shape the community structure of fishery species in the Bohai 
Sea. Although this study didn't include environmental and climate data, however, such results are important for fisheries assessment and maintaining sustainable fisheries management initiative. Moreover, further studies on the fishery community patterns based on climate and environmental observations are needed to verify this outcome.

Acknowledgments. The first author would like to express appreciation to the Chinese Scholarship Council (CSC) for sponsorship during his doctoral degree (CSC No. 2016GXY026). This work supported by the National Basic Research Program of China (No. 2015CB453303). The first author is very thankful and indebted to lab mates from both the Yellow Sea Fisheries Research Institute, Chinese Academy of Fishery Sciences and Laboratory of Fish Population Dynamics, Ocean University of China.

\section{REFERENCES}

[1] Anderson, M. J., Gorley, R. N., Clarke, K. R. (2008): PREMANOVA+ for PRIMER: Guide to software and statistical methods. - PRIMER-E, Plymouth, UK: 1-214.

[2] Aschan, M., Fossheim, M., Greenacre, M., Primicerio, R. (2013): Change in fish community structure in the Barents Sea. - PLoS ONE 8(4): e62748.

[3] Chen, D., Liu, Q., Zeng, X., Su, Z. (1997): Catch composition and seasonal variation of setnet fisheries in the Yellow and Bohai Seas. - Fisheries Research 32: 61-68.

[4] Chen, Y. L., Shan, X., Jin, X., Johannessen, A., Yang, T., Dai, F. (2018): Change in fish diversity and community in the central and southern Yellow Sea from 2003 to 2015. Chinese Journal of Oceanology and Limnology 36(3): 805-817.

[5] Clarke, R. K., Gorley, R. N. (2015): PRIMER 7; User Manual/Tutorial. - PRIMER-E Ltd, Plymouth, UK.

[6] Cui, Y., Chen, B., Chen, J. (2005): Evaluation on self-pollution of marine culture in the Yellow Sea and Bohai Sea. - Chinese Journal of Applied Ecology 16: 180-185. (In Chinese).

[7] Deng, J., Jin, X. (2001): Dynamic characteristics of abundance and community structure of fishery species in the overwintering ground of the Bohai Sea. - Journal of Nature Resource 16: 42-46.

[8] Gao, X., Zhou, F., Chen, C. (2014): Pollution status of the Bohai Sea: An overview of the environmental quality assessment related trace metals. - Environment International 62: 1230 .

[9] Guo, J., Liu, X., Xie, Q. (2013): Characteristics of the Bohai Sea oil spill and its impact on the Bohai Sea ecosystem. - Chinese Science Bulletin 58(19): 2276-2281.

[10] Hu, L. M., Guo, Z. G., Shi, X. F., Qin, Y. W., Lei, K., Zhang, G. (2011): Temporal trends of aliphatic and polyaromatic hydrocarbons in the Bohai Sea, China: Evidence from the sedimentary record. - Organic Geochemistry 42: 1181-1193.

[11] Jin, X., Xu, B., Tang, Q. (2003): Fish assemblage structure in the East China Sea and southern Yellow Sea during autumn and spring. - Journal of Fish Biology 62: 1194-1205.

[12] Jin, X. S. (2004): Long-term changes in fish community structure in the Bohai Sea, China. - Estuarine Coastal and Shelf Science 59: 163-171.

[13] Jin, X. S., Shan, X. J., Li, X. S., Wang, J., Cui, Y., Zuo, T. (2013): Long-term changes of fishery ecosystem in the Laizhou Bay. Science in China. - Earth Science 56: 366-374.

[14] Lee, J.B., Lee, J.H., Shin, Y.J., Zhang, C.I., Cha, H.K. (2010): Seasonal variation of fisheries resources composition in the coastal ecosystem of the middle Yellow Sea of Korea. - Journal of the Korean Society of Fisheries and Ocean Technology 46 (2):126-138 (In Korean). 
[15] Li, X. S., Yu, Z. H., Sun, S., Jin, X. S. (2013): Ecological niche breadth and niche overlap of dominant species of fish assemblage in Yangtze River estuary and its adjacent waters. Chinese Journal of Applied Ecology 24: 2353-2359.

[16] Li, L., Cui, J., Liu, J., Gao, J., Bai, Y., Shi, X. (2016): Extensive study of potential harmful elements ( $\mathrm{Ag}, \mathrm{As}, \mathrm{Hg}, \mathrm{Sb}$, and $\mathrm{Se}$ ) in surface sediments of the Bohai Sea, China: Sources and environmental risks. - Environmental Pollution 219: 432-439.

[17] Lin, C., Su, J., Xu, B., Tang, Q. (2001): Long-term variations of temperature and salinity of the Bohai Sea and their influence on its ecosystem. - Progress in Oceanography 49(1): 7-19.

[18] Liu, X. (1990): Investigations and divisions of fishery resources in the Yellow Sea and Bohai Sea. - Ocean Press, Beijing, 295 pp. (In Chinese).

[19] Liu, X., Wu, J., Han, G. (eds.) (1990): The fisheries resources investigation and division of Yellow and Bohai Seas. - Ocean Press, Beijing, 295 pp.

[20] Liu, S., Lou, S., Kuang, C., Huang, W., Chen, W., Zhang, J. (2011): Water quality assessment by pollution-index method in the coastal waters of Hebei Province in western Bohai Sea, China. - Marine Pollution Bulletin 62(10): 2220-2229.

[21] Liu, Q., Zhang, Q. (2013): Analysis on long-term change of sea surface temperature in the China seas. - Journal of Ocean University of China (Oceanic and Coastal Sea Research) 12(2): 295-300.

[22] Liu, Y. Z., Shen, Y. L., Lv, X. Q., Liu, Q. (2017): Numeric modelling and risk assessment of pollutions in the Chinese Bohai Sea. - Science China Earth Sciences 60(8): 1546-1557.

[23] Ning, X., Lin, C., Su, J., Liu, C., Hao, Q., Le, F., Tang, Q. (2010): Long-term environmental changes and the responses of the ecosystems in the Bohai Sea during 1960-1996. - DeepSea Research Part II Topical Studies in Oceanography 57: 1079-1091.

[24] Pelling, H. E., Uehara, K., Green, J. A. M. (2013): The impact of rapid coastline changes and sea level rise on the tides in the Bohai Sea. - Journal of Geophysical Research: Oceans 118(7): 3462-3472.

[25] Perry, R. I., Cury, P., Brander, K., Jennings, S., Mollmann, C., Planque, B. (2010): Sensitivity of marine systems to climate and fishing: Concepts, issues and management responses. - Journal of Marine Systems 79: 427-435.

[26] Planque, B., Fromentin, J. M., Cury, P., Drinkwater, K. F., Jennings, S., Perry, R. I., Kifani, S. (2010): How does fishing alter marine populations and ecosystem sensitivity to climate? - Journal of Marine Systems 79(3-4): 403-417.

[27] Rahman, M. F., Lin, Q., Shan, X. J., Chen, Y. L., Ding, X. S., Qun, L. (2019): Temporal changes of structure and functioning of the Bohai Sea ecosystem: Insights from Ecopath models. - Thalassas: An International Journal of Marine Sciences 35(2): 625-641.

[28] Rocha, J., Yletyinen, J., Biggs, R., Blenckner, T., Peterson, G. (2015): Marine regime shifts: drivers and impacts on ecosystems services. - Philosophical Transactions Royal Society B 370: 20130273.

[29] Shan, X. J., Jin, X., Wei, Y. (2010a): Taxonomic diversity of fish assemblages in the Changjiang Estuary and its adjacent waters. - Acta Oceanologica Sinica 29(2): 70-80.

[30] Shan, X. J., Jin, X., Wei, Y. (2010b): Fish assemblage structure in the hypoxic zone in the Changjian (Yangtze River) estuary and its adjacent waters. - Chinese Journal of Oceanology and Limnology 28(3): 459-469.

[31] Shan, X. J., Jin, X., Zhou, Z., Dai, F. (2011): Fish community diversity in the middle continental shelf of the East China Sea. - Chinese Journal of Oceanology and Limnology 29(6): 1199-1208

[32] Shan, X. J., Jin, X., Li, Z., Chen, Y., Dai, F. (2012): Fish community structure and stock dynamics of main releasing fish species in the Bohai Sea. - Progress in Fishery Sciences 33: $1-9$. 
[33] Shan, X. J., Sun, P., Jin, X., Li, X., Dai, F. (2013): Long-term changes in fish assemblage structure in the Yellow River ecosystem, China. - Marine and Coastal Fisheries: Dynamics, Management, and Ecosystem Science 5: 65-78.

[34] Shan, X. J., Jin, X., Dai, F., Chen, Y., Yang, T., Yao, J. (2016): Population dynamics of fish species in a marine ecosystem: A case study in the Bohai Sea, China. - Marine and Coastal Fisheries: Dynamics, Management, and Ecosystem Science 8: 100-117.

[35] Song, J., Duan, L. (2018): The Bohai Sea. - In: Sheppard, C. (ed.) World Seas: An Environmental Evaluation. Volume II: The Indian Ocean to the Pacific. Chapter 17, 2nd Edition, 5th September, 932 pp.

[36] Stige, L. C., Ottersen, G., Brander, K., Chan, K. S., Stenseth, N. C. (2006): Cod and climate: effect of the Nort Atlantic oscillation on recruitment in the North Atlantic. - Marine Ecology Progress Series 325: 227-241.

[37] Tang, Q., Jin, X., Wang, J., Zhuang, Z., Cui, Y., Meng, T. (2003): Decadal scale variations of ecosystem productivity and control mechanisms in the Bohai Sea. - Fisheries Oceanography 12: 223-233.

[38] Tang, Q., Su, J., Zhang, J. (2010): China GLOBEC II: a case study of the Yellow Sea and East China Sea ecosystem dynamics. - Deep-Sea Research, Part II 57: 993-995.

[39] Wang, H., Yang, Z., Saito, Y., Liu, J., Sun, X. (2006): Interannual and seasonal variation of the Huanghe (Yellow River) water discharge over the past 50 years: connections to impacts from ENSO events and dams. - Global and Planetary Change 50: 212-225.

[40] Wang, A. Y., Wan, R. J., Jin, X. S. (2010): Decadal variations of ichthyoplankton biodiversity in spring in Laizhou Bay of the Bohai Sea. - Progress in Fishery Science 31: 19-24.

[41] Wu, Q., Wang, J., Li, Z., Chen, R., Sun, J., Jin, X. (2012): Spatial variation of crustacean community structure in Yellow Sea and Bohai Sea in Spring. - Journal of Fisheries of China 36(11): 1685.

[42] Wu, J. Y., Xue, Y., Liu, X. X., Ren, Y. P., Wan, R. (2017): Long-term trends in the mean trophic level of marine fisheries in the Yellow Sea and Bohai Sea. - Periodical of Ocean University of China 47(11): 53-60. (In Chinese).

[43] Xia, J., Xiong, X. J. (2013): Distributions and Seasonal Changes of Water Temperature in the Bohai Sea, Yellow Sea and East China Sea. - Advances in Marine Science 31(1): 5568.

[44] Xu, B., Jin, X. S. (2005): Variations in fish community structure during winter in the southern Yellow Sea over the period 1985-2002. - Fisheries Research 71: 79-91.

[45] Yan, H. K., Wang, N., Yu, T. L., Fu, Q., Liang, C. (2013): Comparing effects of land reclamation techniques on water pollution and fishery loss for a large-scale offshore airport island in Jinzhou Bay, Bohai Sea, China. - Marine Pollution Bulletin 71(1-2): 29-40.

[46] Yu, H. M., Bao, X. W., Lu, C. L., Chen, X., Kuang, L. (2009): Analyses of the log-term salinity variability in the Bohai Sea and the northern Huanghai (Yellow) Sea. - Acta Oceanology Sinica 28(5): 1-8.

[47] Zhang, Y. G., Guan, W., Li, C. P., Dong, L. J. (2002): A study on the exploition and the sustainable utilization of marine resources in the Bohai Sea. - Journal of Natural Resources 17(6): 768-775. (In Chinese).

[48] Zhang, B., Tang, Q. (2004): Study on trophic level of important resource species at high trophic levels in the Bohai Sea, Yellow Sea, and East China Sea. - Advances in Marine Sciences 22: 393-404.

[49] Zhang, Z., Zhu, M., Wang, Z., Wang, J. (2006): Monitoring and managing pollution load in Bohai Sea PR China. - Ocean \& Coastal Management 49(9-10): 706-716.

[50] Zhang, B., Li, Z., Jin, X. (2012): Functional groups of fish assemblages and their major species in the Bohai Sea. - Journal of Fisheries of China 36: 64-72. 
[51] Zhao, Z., Kong, L. (2000): Environmental status quo and protection countermeasures in Bohai marine areas. - Research of Marine Science 13(2): 23-27.

[52] Zhou, M., Yu, R., Meryl, W. (2013): Coastal eutrophication and its associated ecological and environmental problems. - In: Ecosystem issues and policy options addressing the sustainable development of China's ocean and coasts. China Council for International Cooperation on Environment and Development. China Environmental Press, Beijing. 47$61 \mathrm{pp}$.

[53] Zhu, X. H., Tang, Q. S. (2002): Structuring dominant components within fish community in Bohai Sea ecosystem. - Studia Marina Sinica 44: 159-168. 